This value is much larger than the observed total surface area. If this value is divided by the total surface area, the number of boehmite layers can be calculated to be 3.8 , from which the thickness of the crystallite is calculated to be $44 \AA$. This value is in good agreement with the crystallite size determined from the Scherrer equation $(50 \AA)$. Therefore, we concluded that the micropores come from interstices between crystallites. As the thickness of the platelike crystals, which form the honeycomb-like texture, was 500-2000 $\AA$, defects in stacking the boehmite layers with the openings in micropore range ( $\leq 20 \AA)$ are present in the platelike crystals. Moreover, careful examination of the molecular geometry (Figure 7) revealed that gaps between the boehmite layers in the product are too narrow to be accessed by nitrogen molecules, although they may be large enough for water molecules to access the internal surface because hydrogen bondings can be formed. Details of the pore textures of the product and of the aluminas derived from the product will be discussed separately.

Registry No. Water, 7732-18-5; acetaldehyde, 75-07-0; ethanol, 6417-5; acetone, 67-64-1; ethyl acetate, 141-78-6; nitrogen, 7727-37-9.

Contribution from the Central Research and Development Department, ${ }^{\dagger}$ Experimental Station,

E. I. du Pont de Nemours and Company, Wilmington, Delaware 19898, Department of Chemistry, University of New Mexico, Albuquerque, New Mexico 87131, and National Synchrotron Light Source, Brookhaven National Laboratory, Upton, New York 11973

\title{
Characterization of Se-Loaded Molecular Sieves A, X, Y, AlPO-5, and Mordenite
}

\author{
John B. Parise, ${ }^{* \ddagger}$ James E. MacDougall, ${ }^{\ddagger}$ Norman Herron, ${ }^{\ddagger}$ Rod Farlee, ${ }^{\ddagger}$ Arthur W. Sleight, ${ }^{\ddagger}$ \\ Ying Wang, ${ }^{\ddagger}$ Thomas Bein, ${ }^{8}$ Karin Moller, ${ }^{8}$ and Lee M. Moroney" \\ Received January 14, 1987 \\ Selenium has been successfully loaded into molecular sieves A, X, Y, AlPO-5, and mordenite, and the products were characterized \\ by using EXAFS, solid-state NMR, and diffuse-reflectance techniques. This study reveals selenium is predominantly of the trigonal \\ (helical chains) form in all but the A sample, where only the $\mathrm{Se}_{8}$-crown ring form is found. A mixture of allotropes and helical \\ chains occupy the large 3D-pore and channel systems of molecular sieves X and Y; however, a single, probably fixed-pitch \\ helical-chain allotrope occupies the more constrained 12-membered-ring channels found in mordenite and AlPO-5. The high degree \\ of order in these last two sieves is reflected in a strong second-shell feature in the EXAFS spectra.
}

\section{Introduction}

Because of their restricted pore and channel geometries, molecular sieves are potentially useful for the stabilization of unusual chemical species and finely dispersed bulk materials. Particularly, the effect of semiconductor particle size on electronic, optical, and photocatalytic properties is of great interest. Our interest is in the characterization of semiconductors that are in a size range (10-50 $\AA$ ) where they neither exhibit semiconductor bulk properties nor show molecular or atomic properties. This "size quantization" effect has been an intense area of study and discussion recently. ${ }^{1}$ Molecular sieves, which impose size and geometry constraints on encapsulated materials, yield novel environments for studying this phenomenon.

Classically the characterization of the structure of materials absorbed in molecular sieves has been carried out by using the analysis of the three-dimensional (3D) crystallographic data. However, if the absorbed species is not commensurate with the host lattice, this technique is limited; spectroscopic techniques, sensitive to local structure, prove more useful. In this report, we present results of a study of selenium-loaded molecular sieves (mordenite, AlPO-5, A, X, and Y). Free selenium exists in several modifications: a trigonal (t-Se) form containing helical chains, ${ }^{2}$ three monoclinic forms $\left(\alpha, \beta\right.$, and $\gamma$ ) containing $\mathrm{Se}_{8}$ rings, ${ }^{3-6}$ a rhombohedral form containing $\mathrm{Se}_{6}$ rings, ${ }^{7}$ and possibly an orthorhombic form containing $\mathrm{Se}_{7}$ rings. $^{8}$ Only the trigonal form is thermodynamically stable at room temperature and pressure. Upon absorption into a molecular sieve matrix, any of these forms (or a combination of these forms) may be adopted. Extended X-ray absorption fine structure (EXAFS), ${ }^{9}$ optical absorption, and magic angle spinning solid-state nuclear magnetic resonance (MASNMR) have been used to characterize the selenium-loaded molecular sieves. The study was initiated to try to distinguish differences between the forms taken by selenium in the various

\footnotetext{
* To whom correspondence should be addressed at the Department of Inorganic Chemistry, University of Sydney, Sydney, NSW 2006, Australia + Contribution No. 4281.

tE. I. du Pont de Nemours and Company.

University of New Mexico.

"Brookhaven National Laboratory.
}

Table I

\begin{tabular}{llccc}
\hline & & \multicolumn{2}{c}{ chem anal. } & sample \\
\cline { 3 - 5 } sample & source & $\begin{array}{c}\text { wt \% } \% \\
\mathrm{Al}\end{array}$ & $\begin{array}{c}\text { Se } \\
\text { treatment time, } \\
\text { h/temp, }{ }^{\circ} \mathrm{C}\end{array}$ \\
\hline Se-M-9 & $\begin{array}{c}\text { Grande-Paroise } \\
\text { (France) }\end{array}$ & 4.63 & 8.64 & $3 / 150$ \\
Se-AlPO5-15 & $\begin{array}{c}\text { AlPO-5 } \\
\text { (Linde) }\end{array}$ & 17.55 & 15.07 & $2 / 150$ \\
Se-A-6 & $\begin{array}{c}\text { zeolite 5A } \\
\text { (Linde) }\end{array}$ & 15.97 & 6.09 & $8 / 150$ \\
Se-X-26 & $\begin{array}{c}\text { Linde-13X } \\
\text { Se-Y-34 }\end{array}$ & 9.65 & 26.20 & $5 / 200$ \\
Linde-LZY-52 & 5.68 & 33.84 & $3 / 130$
\end{tabular}

sieves and to correlate this with the bulk properties.

To date no three-dimensional analysis of data collected on Se-loaded molecular sieves has been published, although in a study of sodium zeolite A loaded with $23 \mathrm{wt} \%$ sulfur, Seff $^{10}$ found two equivalent $S_{8}$ rings in the crown conformation occupying the large cavity of this molecular sieve. Recently, EXAFS and optical absorbance spectra have been reported for selenium-loaded mordenite. ${ }^{11}$ The existence of single chains of Se in the essentially one-dimensional (1D) pore system of this sieve and a shortening of the Se-Se distance by some $2 \%$, as compared to that in crystalline (trigonal) selenium, was reported.

\section{Experimental Section}

Sample Nomenclature. The samples are designated according to the scheme Se-MS-WT, where $\mathrm{Se}=$ selenium, MS = molecular sieve type, and $\mathrm{WT}=$ weight percent of selenium taken up. For example, Se-

(1) Brus, L. E. J. Phys. Chem. 1986, 90, 2555

(2) Cherin, P.; Unger, P. Inorg. Chem. 1978, 6, 1589.

(3) Cherin, P.; Unger, P. Acta Crystallogr., Sect. B: Struct. Crystallogr. Cryst. Chem. 1972, B28, 313-317.

(4) Burbank, R. D. Acta Crystallogr. 1952, 5, 236.

(5) Marsh, R. E.; Pauling, L.; McCullough, J. D. Acta Crystallogr. 1953, 6,71 .

(6) Foss, O.; Janickis, V. J. Chem. Soc., Dalton Trans. 1980, 624-627.

(7) Miyamoto, Y. Jpn. J. Appl. Phys. 1980, 19, 1813-1819.

(8) Takahashi, T.; Yagi, S.; Sagawa, T.; Nagata, K.; Miyamoto, Y. J. Phys. Soc. Jpn. 1985, 54, 1018-1022.

(9) Lee, P. A.; Citrin, P. H.; Eisenberger, P.; Kincaid, B. M. Rev. Mod. Phys. 1981, 53, 769-803.

(10) Seff, K. J. Phys. Chem. 1972, 18, 2601-2605.

(11) Tamura, K.; Hosokawa, S.; Endo, H.; Yamasaki, S.; Oyanagi, H. J. Phys. Soc. Jpn. 1986, 55, 528. 
AlPO5-15 is selenium-loaded AlPO5 containing $15 \mathrm{wt} \%$ Se. This nomenclature is summarized in Table I.

Sample Preparation. The sieves were all dried at $400^{\circ} \mathrm{C}$ in flowing dry air for $2 \mathrm{~h}$ and allowed to cool under vacuum. A mixture of molecular sieve and selenium was then slowly heated under high vacuum to $\sim 150^{\circ} \mathrm{C}$; this is about $70^{\circ} \mathrm{C}$ below the melting point of selenium. At this temperature the selenium began to sublime, and the material became colored (orange or red, depending on the molecular sieve). The process was continued until the molecular sieve was homogeneous in color and most of the selenium had sublimed. The material was allowed to cool slowly under vacuum $\left(20^{\circ} \mathrm{C} / \mathrm{h}\right)$, taken into a drybox, and sieved to remove excess selenium. Although this process removes bulk selenium, some selenium must be expected to adhere to the surface of the particles of the molecular sieves. However, differences in the spectroscopic properties of these materials (see below) attest to their being different from each other. Samples have shown air sensitivity, by a color change (orange to dark red) and a red shift in their diffuse-reflectance spectra.

Some of the molecular sieves (e.g., zeolite 5A) required much longer times for sorption of the selenium (Table I). In these cases, a static vacuum had to be used to prevent all of the selenium from subliming away from the zeolite before much had been absorbed. To maintain a good static vacuum, the tube was exposed to high vacuum every $1 / 2 \mathrm{~h}$ for several minutes. This procedure gave the highest loadings and the most homogeneously colored materials.

EXAFS Studies. The ability of the EXAFS technique to yield local structural information has been reviewed elsewhere. ${ }^{9}$ Essentially, the technique measures the oscillations observed on the high-energy side of an X-ray absorption edge. The amplitude and frequency of these oscillations are a function of the number and type of neighboring atoms and of the distance of these atoms from the atom involved in the X-ray absorption process.

The normal EXAFS plane wave expression (eq 1) shows the dependence of the fine structure on the various structural parameters. $k$ is

$\chi(k)=\sum_{j} N_{j} / k R_{j}^{2}\left|f_{j}(\pi, k)\right| \times$

$\exp \left(-2 \sigma_{j}^{2} k^{2}\right) \exp \left(-2 R_{j} / \lambda(k)\right) \sin \left(2 k R_{j}+2 \delta_{1}(k)\right)+\arg \left[f_{j}(\pi, k)\right]$

the momentum of the photoelectron ejected in the X-ray absorption process and $\chi$ the normalized oscillations of the fine structure. The frequency of the oscillations varies with $R_{j}$ as well as the term $\delta$ and arg $\left[f_{f}(\pi, k)\right]$, which account for the shift in phase experienced by the ejected photoelectron in passing through the atomic potentials of the central and back-scattering atoms, respectively. By taking the Fourier transform of $\chi$ with respect to $2 k R$, one obtains a function in $R$ space similar to a radial distribution function albeit with the peaks representing the different shells of neighbors shifted $\sim 0.5 \AA$ to low $R$ because of neglect of the phase shift terms in the transform. The amplitude of each successive shell of neighbor contributions to the EXAFS depends on $N_{j}$, the number of atoms in the $j$ th shell, $R_{j}$, the distance of those neighbors from the absorber atom, $\sigma_{j}^{2}$, the variance in that distance, and $\left|f_{j}(\pi, k)\right|$, the back-scattering power of the neighboring atoms. $\sigma_{j}^{2}$ contains contributions from thermally induced vibrational displacements from the perfect interatomic distance plus static displacements within a shell of neighbors.

This second point is of some importance in the case of Se-loaded molecular sieves. The distribution of next-nearest neighbors in the various allotropes of selenium is quite distinctive; only the eight-membered-ring form has a broad distribution of second-nearest-neighbor distances ${ }^{3-6}$ while the trigonal (helical chain) and six-membered-ring forms $^{2-7}$ have only one such distance in the bulk crystalline form. Along with MASNMR and optical evidence, it was hoped this feature could help to indicate which particular allotrope is predominant in the various samples studied.

For collection of EXAFS data, samples were intimately mixed with boron nitride and wax in an oxygen-free glovebox and placed in sample holders between two pieces of Kapton tape. The samples were mounted in a liquid-nitrogen cryostat with Kapton windows.

EXAFS spectra were measured by using the X11-A station on the National Synchrotron Light Source (NSLS) at the Brookhaven National Laboratory (BNL), where typical beam conditions were $2.5 \mathrm{GeV}$ and 100 $\mathrm{mA}$. Data were obtained at the Se K edge $(12657.9 \mathrm{eV})$, and the background function $\left(\mu_{0}\right)$, attributable to absorption from selenium in the absence of neighbors, was determined by using the criteria outlined by Cook and Sayers. ${ }^{12}$ This function was then used to separate the EXAFS function $\chi(k)$ from the absorption spectrum $(\mu)$ by using the expression $\chi=\left(\mu-\mu_{0}\right) / \mu_{0}$.

NMR Studies. Solid-state NMR spectra were measured on a Bruker CXP-300 spectrometer. ${ }^{77} \mathrm{Se}$ NMR poses some experimental difficulties:

(12) Cook, J. W., Jr.; Sayers, D. E. J. Appl. Phys, 1981, 52, 5024-5031. (i) The chemical shift range of ${ }^{~ 17} \mathrm{Se}$ spans about $2500 \mathrm{ppm}^{13}(140 \mathrm{kHz}$ at the 7-T field of the CXP-300 instrument). It is difficult to obtain spectra covering the full chemical shift range. (ii) This value also provides an approximate upper bound on the magnitude of the chemical shift anisotropy that Se might exhibit in a highly asymmetric bonding environment; a resonance this broad would be very difficult to observe. The chemical shift anisotropy of elemental selenium at room temperature is $493 \mathrm{ppm},{ }^{14}$ or $25 \mathrm{kHz}$. A single crystal yields a line width of ca. 250 $\mathrm{Hz}^{14}$ In the MAS spectrum of polycrystalline Se, we therefore expect the intensity to be distributed in six to eight spinning sidebands, the width of each approaching 4 or $5 \mathrm{ppm}$. (iii) The spin-lattice relaxation time of crystalline selenium is long, about $400 \mathrm{~s}$ at room temperature. ${ }^{14}$ The dominant relaxation process is scattering of lattice phonons (Raman process). It is facilitated above $350 \mathrm{~K}$ by thermal population of the conduction band (band gap $1.98 \mathrm{eV}$ for t-Se), but this is not significant at room temperature. The atomic diffusion rate in crystalline Se is negligible at room temperature ${ }^{14}$ (jump rate ca. 0.0001 jumps per second). (iv) Reported ${ }^{77} \mathrm{Se}$ chemical shifts are inconsistent and contain large errors. When this is combined with the large "Se chemical shift range, it makes it difficult to locate and reproduce previous work.

${ }^{77} \mathrm{Se}$ chemical shifts are referred ${ }^{13}$ to $\mathrm{Me}_{2} \mathrm{Se}$. The chemical shift of elemental Se is reported ${ }^{14}$ to be $-616 \mathrm{ppm}$ relative to aqueous $\mathrm{H}_{2} \mathrm{SeO}_{3}$. Aqueous $\mathrm{H}_{2} \mathrm{SeO}_{3}$ has a shift of 1282 or 1288 ppm relative ${ }^{13,15}$ to $\mathrm{Me}_{2} \mathrm{Se}$, so the predicted shift of $\operatorname{Se}(0)$ is 666 or $672 \mathrm{ppm}$.

A convenient standard for setting up solid-state ${ }^{7} \mathrm{Se}$ NMR is reported ${ }^{16}$ to be $\left(\mathrm{NH}_{4}\right)_{2} \mathrm{SeO}_{4}$, which readily undergoes cross polarization from ${ }^{1} \mathrm{H}$ and can be seen quickly. This is used as a secondary chemical shift standard in this work; the reported shift of 1040 ppm relative to $\mathrm{Me}_{2} \mathrm{Se}$ is used ${ }^{16}$ to calculate all shifts reported here.

Optical Measurements. The optical absorption spectra of the selenium-loaded molecular sieves were calculated from their diffuse-reflectance spectra by using the Kubelka-Munk formalism. ${ }^{17}$ The diffuse-reflectance spectra are recorded on a Cary-14 spectrometer fitted with a two-channel diffuse-reflectance attachment. Eastman Kodak barium sulfate was used as the reflectance standard. Se-loaded samples were diluted with the corresponding molecular sieve to avoid saturation. Absorption due to the molecular sieve is subtracted. The absorption spectra reported here were not corrected for the scattering coefficient of the molecular sieve and, strictly speaking, should be identified with the $F$ function in the Kubelka-Munk formalism. ${ }^{17}$ Our measurements on the scattering coefficients of zeolites show that they are only slightly wavelength dependent.

\section{Results and Discussion}

${ }^{77}$ Se NMR. Initial attempts to locate a ${ }^{77}$ Se resonance were complicated by the fact that the reported absolute resonance frequency ${ }^{13}$ is incorrect. A pure sample of gray trigonal Se was prepared, and the spectrum, obtained without cross polarization, was located at an isotropic chemical shift of $793 \mathrm{ppm}$ (Figure 1a) and could be observed with good sensitivity in $1 \mathrm{~h}$. The sideband pattern is that predicted from the chemical shift anisotropy, but the isotropic shift reported in the literature is considerably in error; the likely cause is incorrectly reported shifts of secondary chemical shift standards.

Spectra of Se-loaded samples were obtained by using sufficient averaging ( 180025 scans each), allowing the observation of elemental Se if it were present at the levels reported by elemental analysis (Table I). ${ }^{77}$ Se NMR signals were observed in only two of the samples.

In Se-AlPO5-15, a strong signal at $849 \mathrm{ppm}$ was observed (Figure $1 \mathrm{~b}$ ). While this was $53 \mathrm{ppm}$ from gray Se, the difference is small given the extreme sensitivity of Se shifts and was comparable in magnitude to solvent and concentration shifts seen in solution. More significant was the absence of spinning sidebands, indicating that the chemical shift anisotropy was much smaller than in bulk Se. This could have resulted either from a more nearly spherically symmetric electronic environment about Se or from motional averaging of the CSA by rapid molecular tumbling

(13) Rodger, C.; Sheppard, N.; McFarlane, H. C. E.; McFarlane, W. In NMR and the Periodic Table; Harris, R. K., Mann, B. E., Eds.; Academic: New York, 1978; Chapter 12.

(14) Guenther, B.; Kanert, O. Phys. Rev. B: Condens. Matter 1985, 31, 20.

(15) Collins, M. J.; Ratcliffe, C. I.; Ripmeester, J. A. J. Magn. Reson. 1986, 68,172 .

(16) Koch, W.; Lutz, O.; Molle, A. Z. Phys. A 1978, 289, 17

(17) Kortum, G. Reflectance Spectroscopy; Springer-Verlag: New York, 1969. 

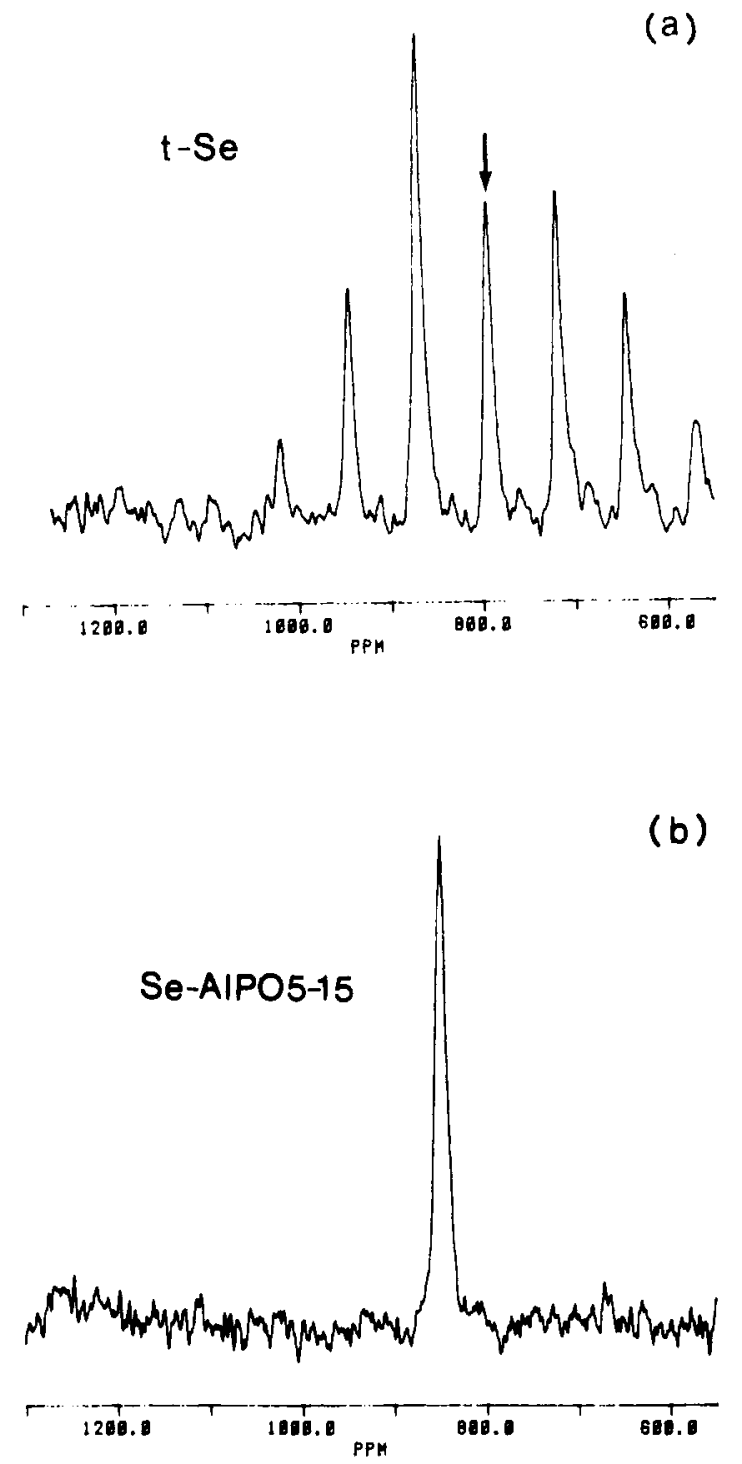

(c)
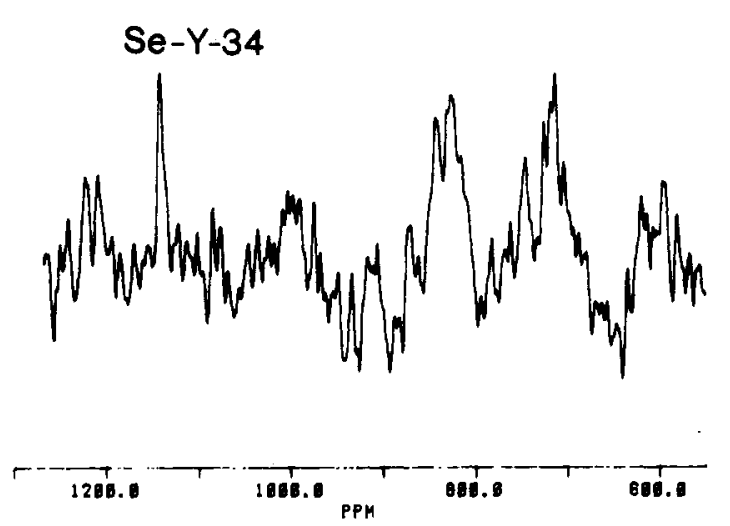

Figure 1. ${ }^{77} \mathrm{Se}$ MASNMR spectra for (a) trigonal Se (t-Se) showing spinning side bands, (b) Se-AlPO5-15 showing a single resonance (849 ppm) close to that for t-Se (a), and (c) Se-Y-34 showing the presence of three resonances attributed to $\mathrm{SeO}_{4}{ }^{2-}(1339 \mathrm{ppm})$ and two forms of $\mathrm{Se}^{0}(824$ and $712 \mathrm{ppm})$.

or diffusion within the molecular sieve. This latter possibility seems unlikely.

In Se-Y-34, three weak ${ }^{77}$ Se resonances were observed (Figure 1c). The two major (albeit weak) lines are at 824 and $712 \mathrm{ppm}$,
Table II. Comparison of Selenium Band Gaps

\begin{tabular}{lcl}
\multicolumn{1}{c}{ Se sample } & band gap, eV & basic unit \\
\hline t-Se (trigonal) & 1.98 & helical chain \\
$\alpha$-Se (monoclinic) & 2.53 & Se $e_{8}$ ring \\
Se-M-9 & 2.25 & \\
Se-AlPO5-15 & 2.4 & \\
Se-A-6 & 2.85 & \\
Se-X-26 & 2.3 & \\
Se-Y-34 & 2.2 &
\end{tabular}

Table III. Peak Positions $(\AA)$ in Se-Loaded Molecular Sieves (Figures 2-6) Obtained from Curve Fitting Using Experimentally Determined Phase Shifts and Amplitude Functions

\begin{tabular}{lcccccc}
\hline \multicolumn{1}{c}{ sample } & $\begin{array}{c}\text { fit } \\
\text { to 1st } \\
\text { shell }^{a}\end{array}$ & \multicolumn{1}{c}{$\begin{array}{c}\text { fit } \\
\text { to } \sigma^{2 b}\end{array}$} & $\begin{array}{c}\text { fo lst } \\
\text { shell }^{c}\end{array}$ & $10^{3} \sigma^{2 b}$ & $\begin{array}{c}\text { fit } \\
\text { to } 2 \text { nd } \\
\text { shell }^{c}\end{array}$ & $10^{3} \sigma^{2 b}$ \\
\hline Se-A-6 & $2.34(1)$ & $4(1)$ & $2.35(1)$ & $5(1)$ & $3.71(1)$ & $11(1)$ \\
Se-M-9 & $2.35(1)$ & $4(1)$ & $2.35(1)$ & $5(1)$ & $3.63(1)$ & $11(1)$ \\
Se-AlPO5-15 & $2.34(1)$ & $3(1)$ & $2.35(1)$ & $5(1)$ & $3.66(1)$ & $10(1)$ \\
Se-X-26 & $2.34(1)$ & $4(1)$ & $2.35(1)$ & $5(1)$ & $3.67(1)$ & $13(1)$ \\
Se-Y-34 & $2.34(1)$ & $4(1)$ & $2.35(1)$ & $5(1)$ & $3.67(1)$ & $12(1)$ \\
t-Se & 2.373 & $7(1)$ & & & & \\
g-Se & $2.35(1)$ & $5(1)$ & & & &
\end{tabular}

${ }^{a}$ With $\mathrm{t}-\mathrm{Se}$ as the standard. The coordination number was fixed at 2. ${ }^{b}$ Data with $k<5 \AA$ excluded. $\sigma$ is the variance of the refined distance related to the Debye-Waller factor. ${ }^{\circ}$ Crystalline As is used to extract amplitude and phase functions. Data $<5 \AA$ were excluded. The coordination number was fixed at 2 .

consistent with two inequivalent $\mathrm{Se}^{0}$ sites. A narrower and weaker line at $1139 \mathrm{ppm}$ is in the range expected for selenates and suggests that some slight oxidation has occurred.

The weakness of the signal in Se-Y-34 is most likely a result of $T_{1} s$ times that are much longer than those of bulk $\mathrm{Se}^{0}$. While an expected relaxation time cannot be predicted, it was hoped that proton dipolar relaxation due to hydroxyls attached to the framework of the molecular sieves might occur. An attempt to observe the signal in Se-A- 6 by using proton cross polarization was also unsuccessful. While the absence of signal could also result from partial oxidation to selenite (which is outside of the window of chemical shifts observed under the conditions used) or from extreme broadening due to disorder, a likely cause is the long $T_{1} s$, and this negative result does not yield any useful structural information on those samples for which no signal is observed. In the case of Se-AlPO5-15 only one signal is observed, implying only one allotrope exists. On the other hand, in Se-Y-34 at least two allotropic forms of Se are present.

Optical Properties of Se-Loaded Molecular Sieves. The absorption spectra of selenium in different zeolites are shown in Figure 2. For the purpose of comparison, the small particle spectra for Se are calculated with the Lorenz-Mie formalism in the electric dipole limit. The bulk optical constants of trigonal Se (helical chain) and monoclinic Se (eight-membered ring) ${ }^{18,19}$ are used in the calculation. These are the spectra one would expect if the bulk optical properties were maintained in the small-particle-size regime $(<100 \AA)$, i.e. if the size effect were absent. The band gap of the selenium-loaded samples was determined by using the formula for the direct allowed transition, $\sigma(h \nu)=A(h \nu-$ $\left.E_{\mathrm{g}}\right)^{1 / 2}$, where $\sigma$ is the absorption coefficient and $E_{\mathrm{g}}$ is the band gap. Figure 3 shows two examples. The results are summarized in Table II, and several conclusions can be derived.

(1) The absorption spectrum of Se in Se-A-6 is drastically different from those in other samples (X, Y, AlPO5, and $M$ ). While subtle differences exist among Se samples in X, Y, AlPO5, and $M$, they are basically similar, and their band-gap energies are all close to that for trigonal Se (Table II). For Se-A-6, the band gap is larger by more than $0.5 \mathrm{eV}$ and is close to the value for monoclinic Se. These data suggest that in X, Y, AlPO5, and $M$ the dominant Se structure is the helical chain of $t-S e$. In

(18) Tutihasi, S.; Chem, I. Phys. Rev. 1967, 158, 623

(19) Dalrymple, R. J. F.; Spear, W. E. J. Phys. Chem. Solids 1972, 33, 197. 


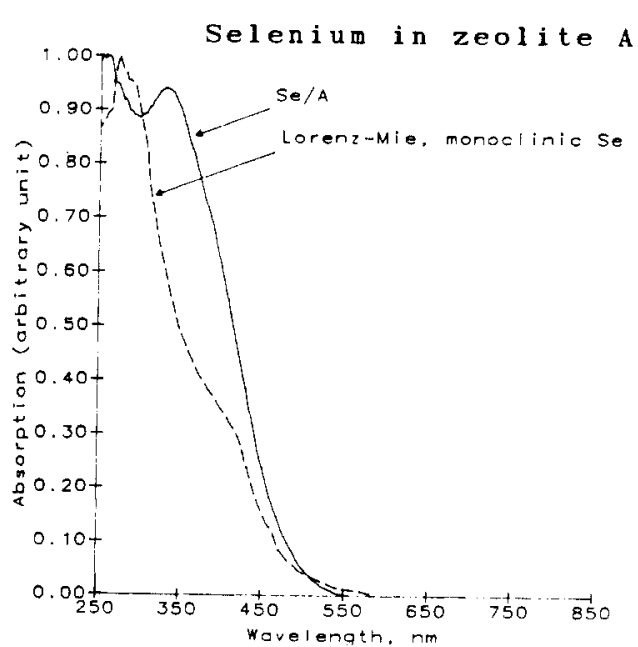

a

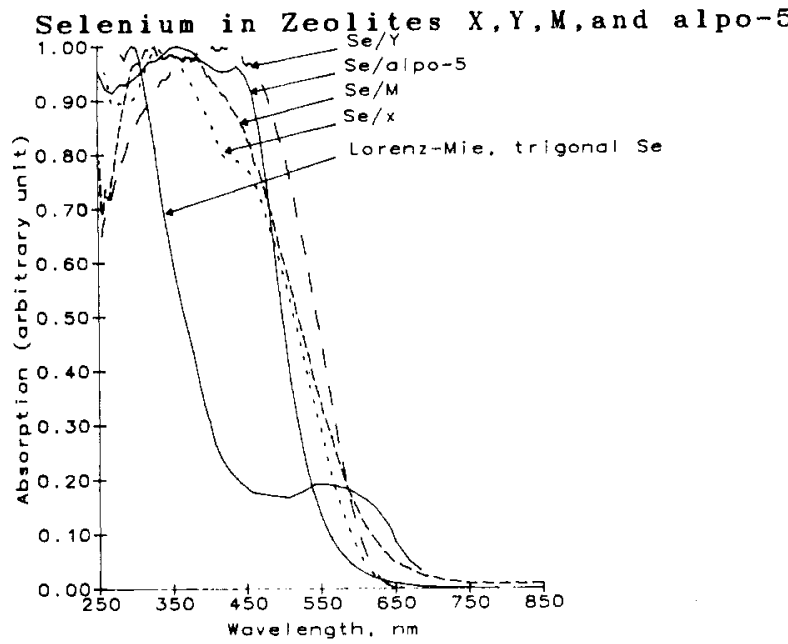

b

Figure 2. Optical absorption spectra for (a) Se-A-6 compared to that calculated for monoclinic (crown-Se ${ }_{8}$-ring form) selenium and (b) all other Se-loaded molecular sieves compared to the calculated spectrum for $\mathrm{t}$-Se. Note the magnitude of absorption is arbitrary. All spectra are normalized.

Se-A-6, the $\mathrm{Se}_{8}$ ring structure dominates. It is possible that a mixture of chain and ring structures exist for $\mathrm{Se}$ in molecular sieves $\mathrm{X}, \mathrm{Y}, \mathrm{AlPO5}$, and $\mathrm{M}$.

(2) The band gaps of $\mathrm{Se}$ in the molecular sieves are blue-shifted from the respective band gaps of bulk trigonal and monoclinic forms. In molecular sieves X, Y, AlPO5, and M, a shift of more than $0.2 \mathrm{eV}$ from the value for trigonal Se is observed (Table II and Figure 1). In Se-A-6, there is a $0.3-\mathrm{eV}$ shift from the value for monoclinic Se. The dependence of band gap on particle size is now well-documented ${ }^{20,21}$ for small semiconductor particles such as $\mathrm{CdS}$. In these isotropic semiconductors the increase in band gap can be attributed mostly to the increase in electron-hole kinetic energy as a result of their spatial confinement. The situation of $\mathrm{Se}$ is quite unique in that subunits exist (helical chain and puckered rings). The bonding within the subunit is strong, while the bonding between the subunits is relatively weak. If in molecular sieves $\mathrm{X}, \mathrm{Y}, \mathrm{AlPO}$, and M only isolated Se chains exist, the blue shift of the band gap can be attributed to the loss of chain-chain interaction and possibly the quantization effect due to reduced chain length. In $\mathrm{Se}-\mathrm{A}-6$, if isolated $\mathrm{Se}_{8}$ rings exist, then the blue shift of the band gap can be attributed to the loss of ring-ring interaction.

(20) Brus, L. E. J. Phys. Chem. 1986, 90, 2555 and references therein

(21) Wang, Y.; Herron, N., submitted for publication.

(22) Breck, D. W. Zeolite Molecular Sieves; Wiley-Interscience: New York 1974.

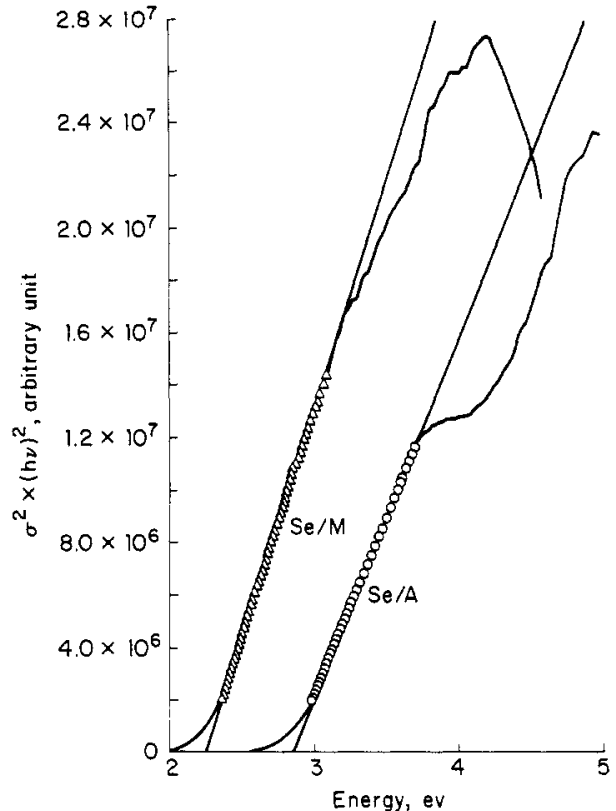

Figure 3. Plots of $\left[\sigma^{2}(h \nu)^{2}\right]$ vs $h \nu$ for Se-A-6 and Se-M-9. The straight lines represent direct, allowed transitions. Band gaps are obtained from the intercepts.

(3) A direct result of the loss of chain-chain and ring-ring interaction for Se in molecular sieves is that the structure of the chain or ring itself may change slightly. The most likely result is the shortening of bond distance and the change in helical pitch. Furthermore, zeolite internal structure can also modulate the Se structure. All these can make small changes in the optical properties of Se and may contribute to the subtle difference observed among the absorption spectra of Se in different zeolites (Figure 2).

EXAFS of Se-Loaded Molecular Sieves. Figures $4 a-8$ a show the $k^{1}$-weighted EXAFS oscillations obtained for the Se K edge of Se-loaded molecular sieves at liquid-nitrogen temperature. The envelopes of the EXAFS oscillations, to a first approximation, are similar to those for trigonal Se (Figure 9a) and glassy selenium (Figure 10a) and reflect the form of the Se back-scattering function. ${ }^{9}$ Thus, the major back-scattering contributions are due to Se-Se neighbors. Fourier transforms (FT's) of these data in the range $1.5 \leq k \leq 20$ are shown in Figures $4 \mathrm{~b}-10 \mathrm{~b}$.

In each FT there are two prominent peaks: one at $2.0 \AA$ and a second in the region about $3.5 \AA$ (Figures $4-10$ ). As previously noted, the radial distances shown in the Fourier transforms are shifted to low $r$ because of phase shift terms in eq 1 . However, they will be used for internal comparison between different samples.

By careful examination and comparison of the EXAFS oscillations from standard materials ( $t-S e$, glassy Se) and Se-loaded molecular sieves, much qualitative information can be inferred without recourse to complicated multiparameter fitting. In the case of crystalline trigonal Se (t-Se) (Figure 9), these peaks correspond to the first-shell Se-Se bond along the helical chain $(2.37 \AA$; at $2.0 \AA$ in FT), to the interchain second shell $(3.44 \AA$; at $3.1 \AA$ in FT), and to the interchain second shell ( $3.72 \AA$; at $3.5 \AA$ in FT). Table IV lists additional interatomic distances for the different allotropes of Se. When the selenium is loaded into the sieves, the peak around $3.1 \AA$ disappears in every case, while that at $3.5 \AA$ is retained (Figures $4 b-9 b$ ). The same feature is noted in glassy selenium (Figure 10b). This observation provides evidence for a loss of interchain correlation by introducing the Se into the sieve.

The dominant contribution above $k=4 \AA^{-1}$ to the second shell is from Se-Se interactions. This can be appreciated by Fourier filtering ${ }^{9}$ the second-shell contribution to the EXAFS. Figure 11 shows the second-shell contribution from Se-AlPO5-15. The profile of the back-scattering envelope, which reveals that backscattering is still strong at high $k$, is evidence that the predominant 

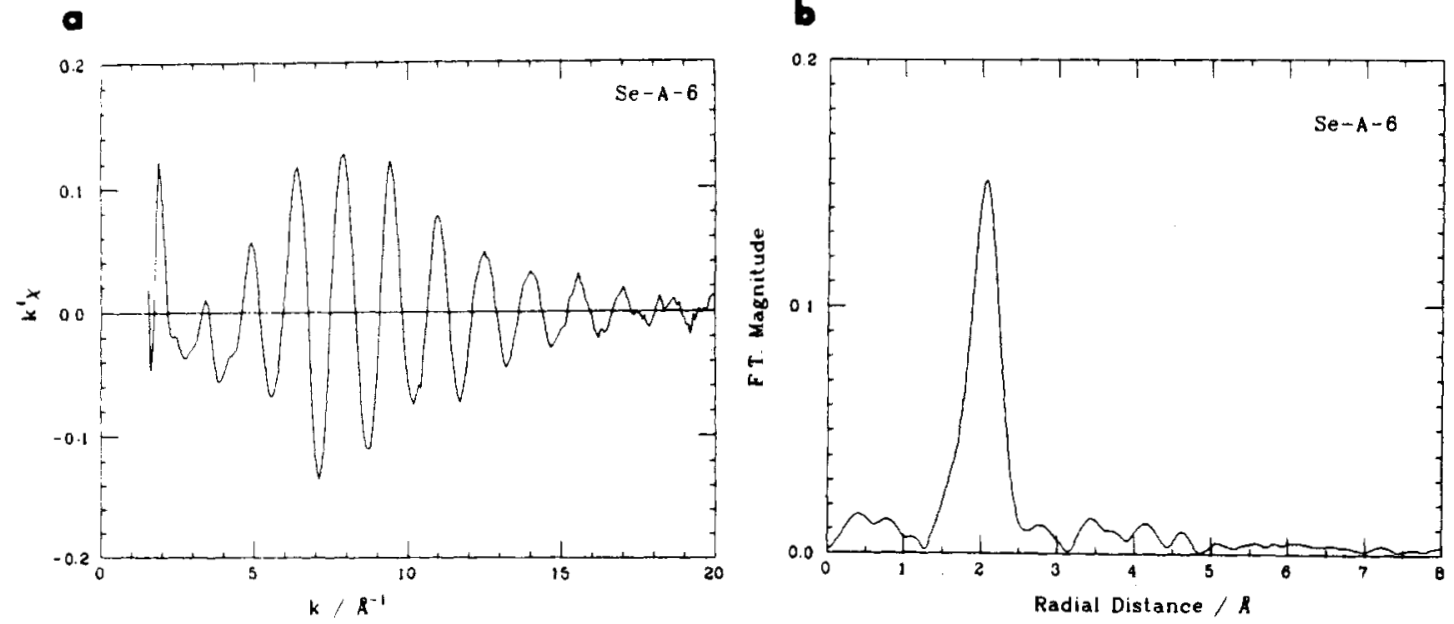

Figure 4. (a) $k$-weighted experimental EXAFS $(\chi)$ of the Se K edge of Se-A-6 (Table 1 ) at $-195^{\circ} \mathrm{C}$. (b) Magnitude of the Fourier transform of data in (a).
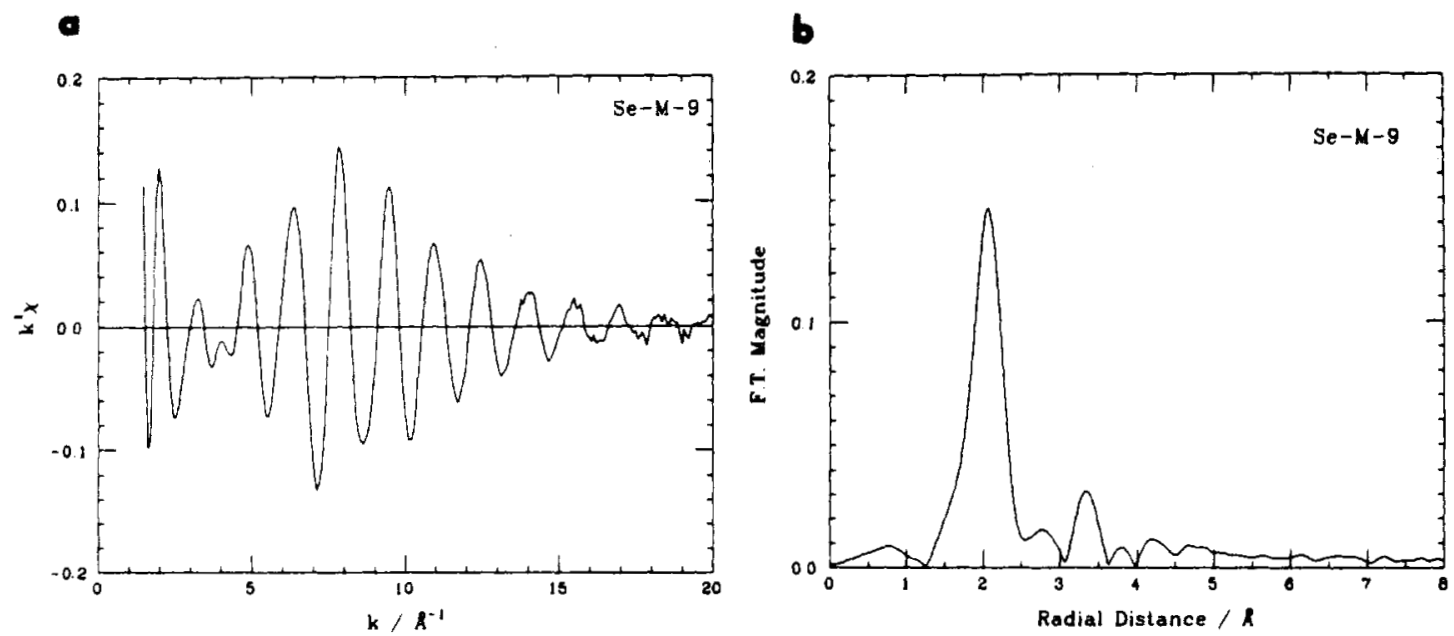

Figure 5. (a) $k$-weighted experimental EXAFS of the Se K edge of Se-M-9 at $-195^{\circ} \mathrm{C}$. (b) Magnitude of the Fourier transform of (a).
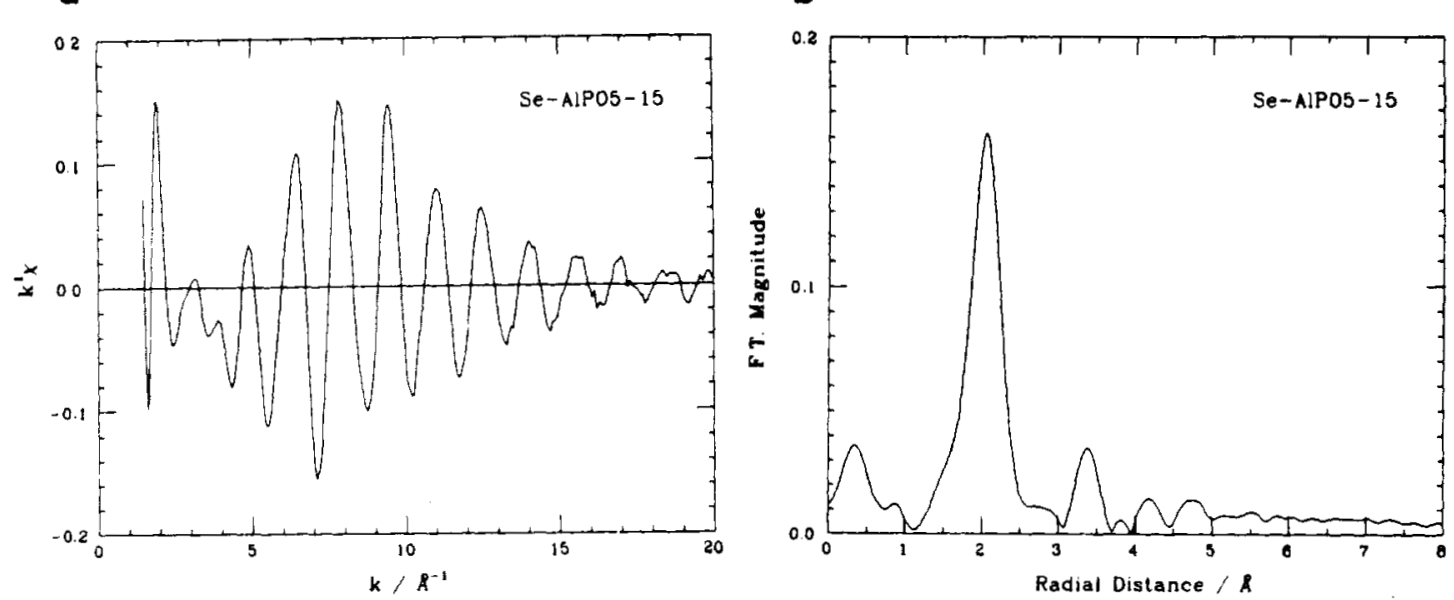

Figure 6. (a) $k$-weighted experimental EXAFS of the Se K edge of Se-AlPO5-15 at $-195^{\circ} \mathrm{C}$. (b) Magnitude of the Fourier transform of (a).

contribution to the second shell is from the Se next-nearest neighbor.

The magnitude of this second-shell peak varies from sample to sample, being greatest in the ID molecular sieves M and AIPO5 (Figures 5 and 6 ) and closer to that observed for glassy Se for $\mathrm{Se}-\mathrm{X}$ and $\mathrm{Se}-\mathrm{Y}$. Consistent with the optical data, the situation for Se-A is somewhat anomalous. The form of the second shell (Figure 4) in this case resembles neither that for t-Se nor that for $\mathrm{g}$-Se but rather has a peak centered at $3.5 \AA$ with a distribution to $3.8 \AA$; the second-shell peaks for other samples are, by com- parison, sharp. A similar distribution is expected for Se-Se next-nearest-neighbor distances in an $\mathrm{Se}_{8}$ ring. ${ }^{6}$ All other allotropes of Se have only one intramolecular next-nearest-neighbor distance (Table IV). This is a further indication of the presence of only $\mathrm{Se}_{8}$ units in Se-A-6, and this may be due to this molecular sieve's small eight-membered-ring windows, which hinder the formation of chain structures.

While comparison of the frequencies of the Fourier-filtered first shell shows that the Se-Se nearest-neighbor distances are very similar for all the Se-loaded molecular sieves, the same is not so 

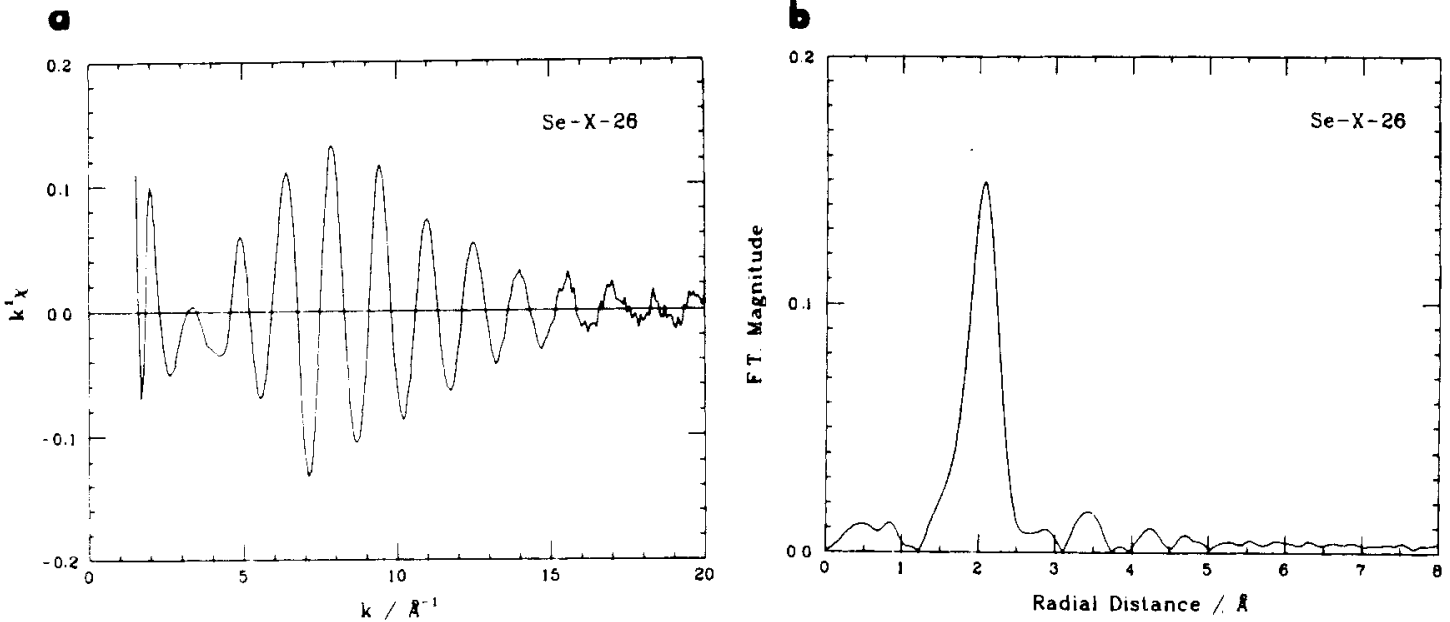

Figure 7. (a) $k$-weighted experimental EXAFS of the Se K edge of Se-X-26 at $-195^{\circ} \mathrm{C}$. (b) Magnitude of the Fourier transform of (a).
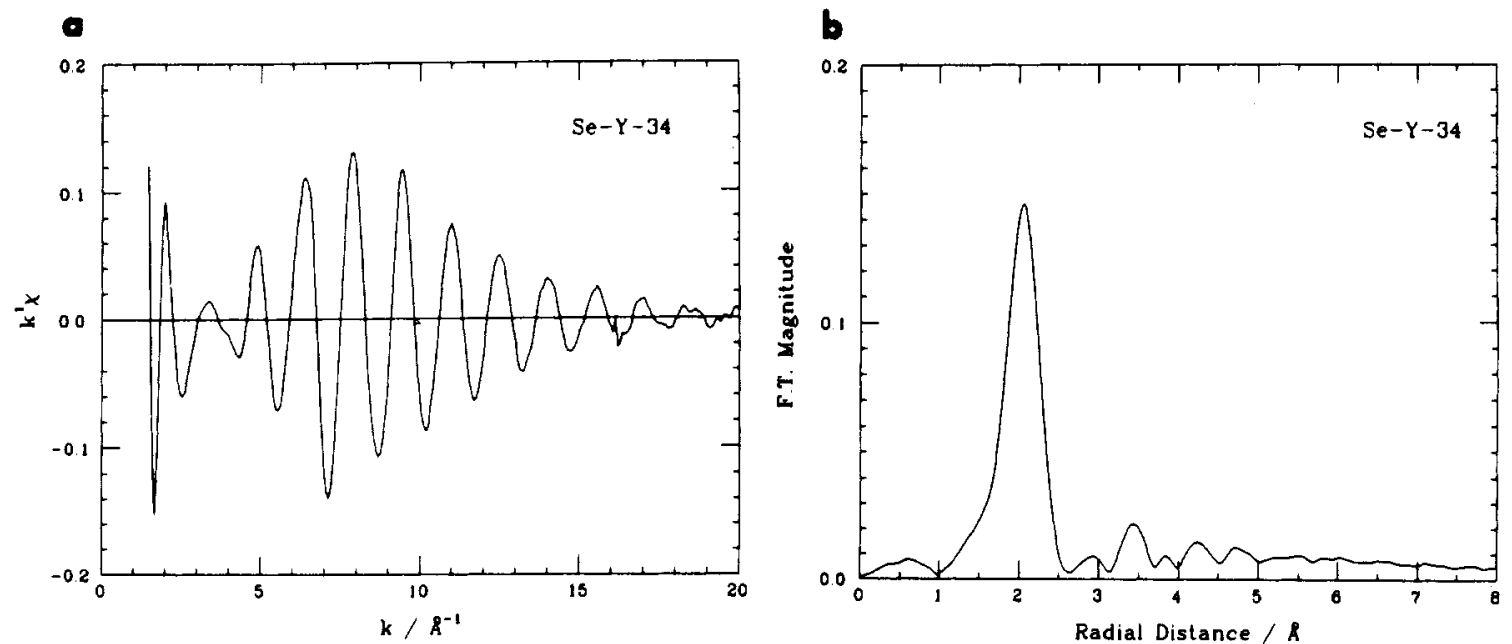

Figure 8. (a) $k$-weighted experimental EXAFS of the Se K edge of Se-Y-34 at $-195^{\circ} \mathrm{C}$. (b) Magnitude of the Fourier transform of (a).
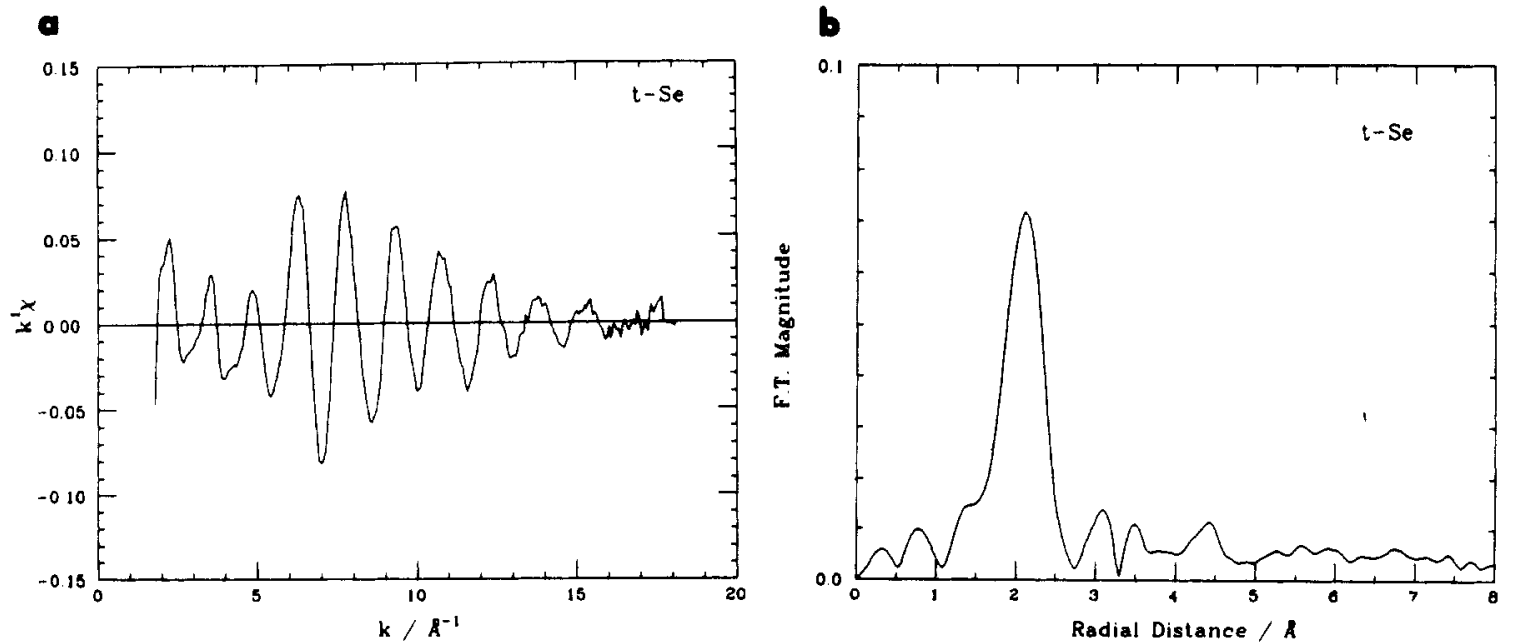

Figure 9. (a) $k$-weighted experimental EXAFS of the Se K edge of trigonal selenium (t-Se) at $-195^{\circ} \mathrm{C}$. (b) Magnitude of the Fourier transform of (a).

for the second-shell contributions. Notably, the amplitude and frequency of the EXAFS due to the second shell for Se-AIPO5-15 and Se-M-9 are almost identical, suggesting the same form of selenium is found in both sieves. On the other hand, the more damped and higher frequency oscillations found in Se-X-36 when compared to those in Se-AlPO5-15 (Figure 12) suggests the forms are different; the average second-shell distance is longer and the variance in this distance greater for selenium in molecular sieve $X$. Qualitatively this agrees with conclusions drawn from the optical and NMR data; Se exists in a trigonal form in M, AlPO5, and $X$. The pore system of the sieve can be expected to impose limitations on the arrangement of the molecular $\mathrm{Se}_{n}$ units within them. In the case of helical chains this is expected to result in isolated single chains in one-dimensional channel systems, i.e. mordenite and AlPO5. However, these constraints on the helical chains are relaxed in the large-pore 3D-channel system of $X$. Although the distributions of second-shell distances in Se-M-9 and Se-AlPO5-15 are similar (the amplitudes in Figure 11), the 

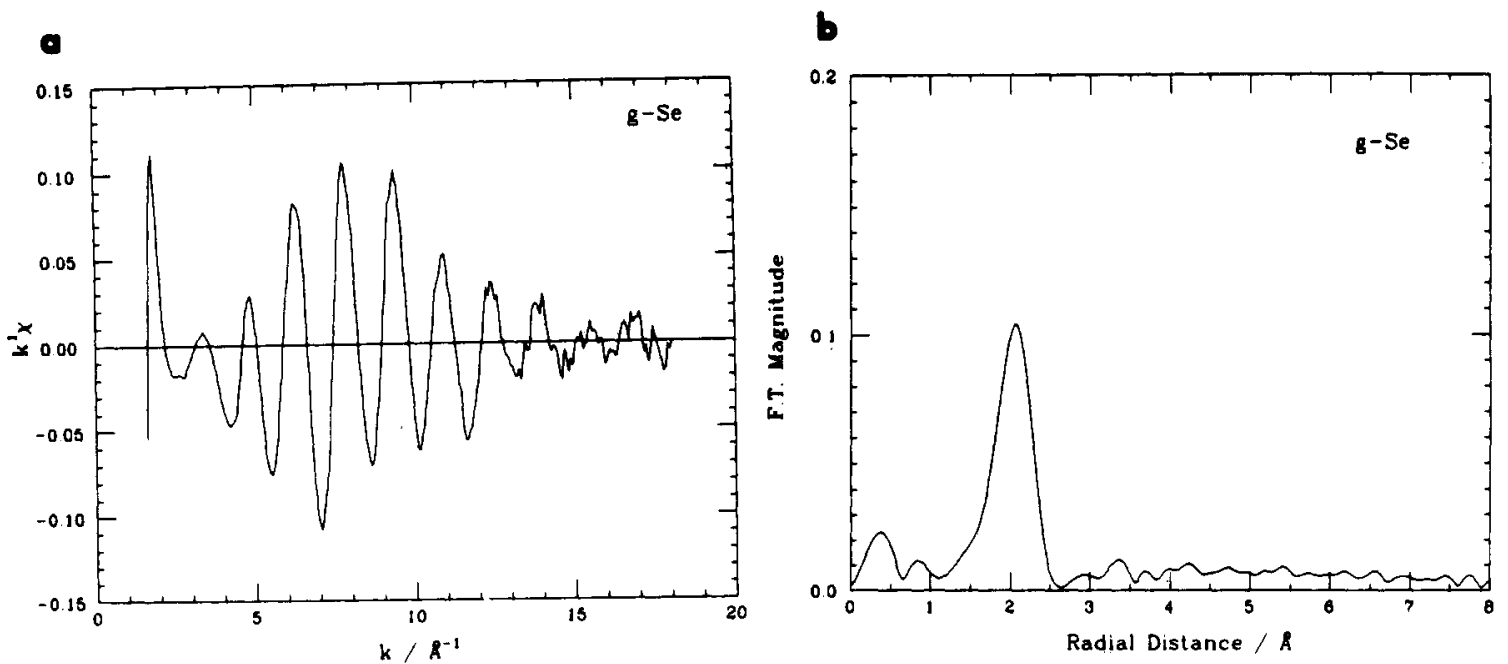

Figure 10. (a) $k$-weighted experimental EXAFS of the Se K edge of glassy selenium (g-Se) at $-195^{\circ} \mathrm{C}$. (b) Magnitude of the Fourier transform of (a).

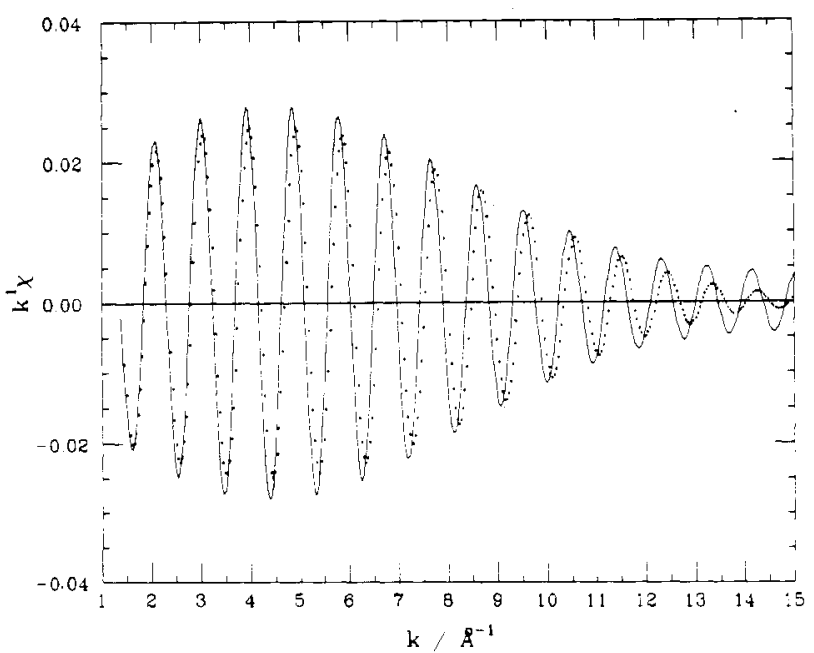

Figure 11. Comparison of Fourier-filtered EXAFS of Se-Se second-shel contributions to the experimental EXAFS for Se-AIPO5-15 (solid line) and Se-M-9 (dotted line) illustrating comparable amplitudes (same degree of order) and a slightly lower frequency for Se-M-9 (shorter radial distance from $\mathrm{Se}$ to its next-nearest neighbors)

Table IV. Selected Interatomic Distances for Various Allotropes of Se

\begin{tabular}{|c|c|c|c|c|}
\hline \multirow[b]{2}{*}{ allotrope } & \multirow{2}{*}{$\begin{array}{l}\text { struct } \\
\text { building } \\
\text { unit }\end{array}$} & \multicolumn{2}{|r|}{$\langle\mathrm{Se}-\mathrm{Se}\rangle, \AA$} & \\
\hline & & $\begin{array}{l}\text { nearest } \\
\text { neighbor }\end{array}$ & $\begin{array}{l}\text { next nearest } \\
\text { neighbors }\end{array}$ & \\
\hline trigonal Se & helical chains & 2.373 & $\begin{array}{l}3.717 \text { (along chain), } \\
3.436 \text { (between chains) }\end{array}$ & 3 \\
\hline$-\mathrm{Se}^{a}$ & 8-ring & 2.318 & 3.71 & \\
\hline$\beta-\mathrm{Se}^{a}$ & 8-ring & 2.337 & 3.71 & \\
\hline$\gamma-\mathrm{Se}^{a}$ & 8-ring & 2.334 & 3.71 & \\
\hline $\mathrm{r}-\mathrm{Se}$ & 6-ring & 2.356 & 3.64 & 7 \\
\hline $\mathrm{O}-\mathrm{Se}^{b}$ & 7-ring & 2.363 & 3.69 & 8 \\
\hline
\end{tabular}

${ }^{a}$ These crown conformation eight-membered-ring forms differ mainly in the way in which rings are packed within the unit cell. ${ }^{b}$ No structural analysis; structure inferred from calculation assuming fixed Se-Se distance.

distances are slightly shorter in the case of Se-M-9.

In order to quantify these observations, it was necessary to determine the function $\delta_{1},(\pi k)$, and $F_{j}(\pi, k)$. These were obtained initially from the experimental data for the structural standard t-Se by using Fourier techniques ${ }^{9}$ and assuming a Se-Se distance ${ }^{3}$ of $2.373 \AA$. From this information, the component frequencies of the EXAFS of the samples may be extracted by Fourier filtering and fitted individually by refining the mean radial distance $\left(R_{j}\right)$ and its variance $\left(\sigma^{2}\right)$, which can be related to the thermal and static

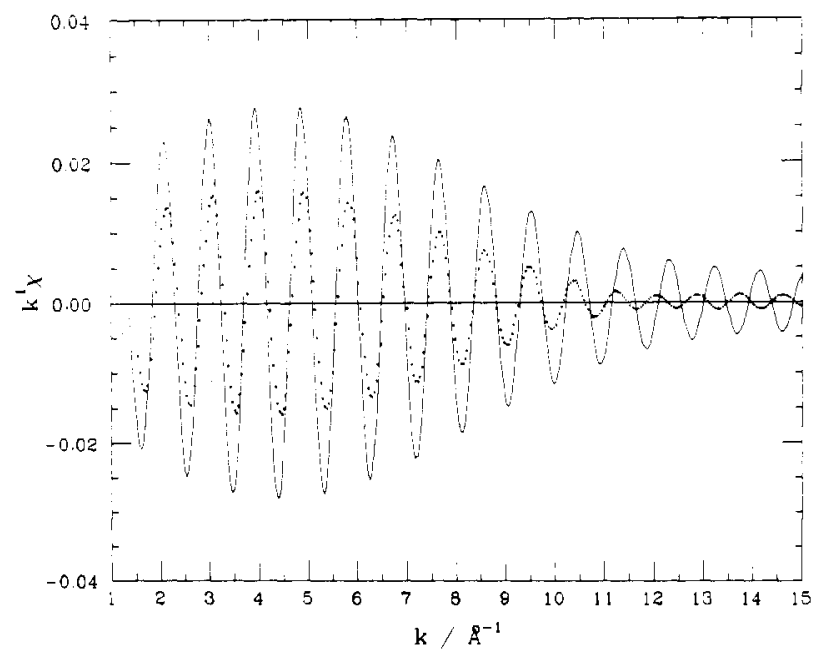

Figure 12. Comparison of Fourier-filtered EXAFS of Se second-shell contributions from Se-AlPO5-15 (solid line) and Se-Y-34 illustrating a much lower amplitude for Se-Y-34, indicating a greater variance in the radial distance to second-shell neighbors.

displacement about this mean radial distance. Because of the difficulties associated with the possibility of Se-framework interactions, several preliminary attempts were made to fit the data by using different fitting strategies. The results are shown in Table III. In order to exclude possible Se-framework interactions, data with $k<5 \AA$ were excluded from the refinement. The backscattering functions for $\mathrm{Si}, \mathrm{Al}$, and $\mathrm{O}$ fall off quickly at high values of $k$. In order to preclude the possibility that deviation from structural homogeneity in the t-Se sample might introduce inaccuracies with its use as a structural standard, we also fitted the data using crystalline arsenic as a standard. Arsenic being adjacent in the periodic table, its scattering behavior is virtually indistinguishable from that of Se. Use of either standard led to identical results (Table III).

Fits to the first shell indicate that Se incorporated into molecular sieves shows a Se-Se distance slightly shorter than that of trigonal, crystalline Se. The distance reduces from $2.373 \AA$ (t-Se) to about $2.34 \AA$ for all molecular sieves and to $2.35 \AA$ for glassy Se. Allotropes of Se containing ring units also have shorter bond distances (between 2.32 and $2.36 \AA$; see Table IV).

The decrease in Se-Se distance observed is less than that reported previously. ${ }^{11}$ This shortening of the Se-Se bond could account for some of the shift to higher energies observed in the optical experiments.

Distances in the second shell show some variation with the mean distance observed for Se-A-6 (3.71 (1) $\AA$ ) consistent with the mean distance found in the eight-membered-ring form of selenium 
(Table IV and ref 6). Second-shell distances for all other samples are shorter than would be expected for a helical chain of trigonal selenium. This could be due to a change in the pitch of the chain, the presence of six-membered rings, or a chain bending back upon itself. In the case of Se-X-34 and Se-Y-26, the breadth of the second-shell peak in the FT along with the optical and NMR results suggests a mixture of at least two forms of selenium. The helical chains present cannot have a regular Se-Se-Se dihedral angle, or we would expect a sharper distribution of next-nearest-neighbor distances and a higher EXAFS amplitude (Figures 6 and 7). The choice of coordination number becomes somewhat arbitrary when attempting to fit one average interatomic distance to data from several atoms at slightly different distances. In this case the coordination number was fixed at 2. Although this is a good approximation in the case of Se-M-9 and Se-AlPO5-15, where single helical chains occupy the channels, the retained Se-Se next-nearest-neighbor distances for Se-X-26, Se-Y-36, and Se-A-6 represent the weighted-average distance for all allotropes and conformations present in the channels of the molecular sieves.

The loading density of Se in Se-M-9 corresponds to about 3.6 Se atoms per unit cell, which, if it were to adopt the helical-chain allotrope exclusively, would correspond to a chain $6 \AA$ in length per unit cell. When this is compared with the $c$ axial length for hydrated mordenite $(7.5 \AA)$, a helical chain would not completely fill the 12-membered-ring channels in mordenite. A similar argument can be extended to Se-AIPO5-15, where 3.5 Se atoms per unit cell corresponds to a chain $5.82 \AA$ in length. Although on the basis of this evidence it is difficult to draw a firm conclusion on the identity of the Se allotrope present, eight-membered rings and bent helical chains must be excluded. The allotrope present is probably a single compressed helical chain in both cases. Interestingly, the $23 \mathrm{wt} \%$ loading of Se in mordenite reported elsewhere ${ }^{11}$ corresponds to $11.6 \mathrm{Se}$ atoms per unit cell or a chain $19 \AA$ in length or two chains $9.55 \AA$ in length and so on.

\section{Conclusion}

The combination of EXAFS and solid-state NMR spectros- copies with optical absorption experiments reveals the effect of spatial confinement of Se in molecular sieves. Encapsulation of $\mathrm{Se}$ in various sieves causes the $\mathrm{Se}-\mathrm{Se}$ bond length to shorten relative to the distance observed in helical chains of trigonal Se. No interchain correlation remains if $\mathrm{Se}$ is absorbed in the different sieves, indicating the presence of isolated, single molecular $\mathrm{Se}_{n}$ units. The analysis of the second-nearest-neighbor shell of Se in the one-dimensional (1D) channel systems of mordenite and AlPO5 indicates the highest degree of order of the $\mathrm{Se}_{n}$ units compared to other sieves. Combined with evidence from NMR experiments with AIPO5, loading densities, and optical absorption data, these results are consistent with the presence of a single, highly ordered helical chain allotrope of Se in AlPO5 and mordenite.

The EXAFS data indicate a less restricted next-nearestneighbor environment in the case of large-pore $X$ and $Y$, consistent with the presence of a variety of conformations or structures. Optical data support this interpretation, and NMR data suggest that two such structures are present with one probably being helical-chain Se. Optical absorption as well as structural results from EXAFS measurements point to the eight-membered ring allotrope exclusively adsorbed into A zeolite.

Clearly, spatial confinement of semiconducting materials in microporous solids offers much potential for the adjustment of such physical properties as band gaps and optical behavior.

Acknowledgment. We thank Steve Heald, John Tranquada, Jack Scrofani, and Larry Fareria for assistance during data collection. Part of this work was supported by DOE Grant No. DE-AS0580ER10742. C. Yang kindly provided EXAFS data for crystalline $\mathrm{Se}$ and $\mathrm{As}$ as well as glassy Se.

Registry No. Se, 7782-49-2; AlPO-5, 98499-64-0; mordenite, $12173-98-7$.

Contribution from the Laboratoire de Chimie Thêorique, Bât. 490, Université de Paris-Sud, 91405 Orsay Cedex, France, and Department of Chemistry, North Carolina State University, Raleigh, North Carolina 27695-8204

\title{
Semiconducting Properties of $\mathrm{Li}_{0.33} \mathrm{MoO}_{3}$
}

\author{
Enric Canadell ${ }^{* 1 a}$ and Myung-Hwan Whangbo*1b
}

Received August 10, 1987

The bottom d-block band electronic structure of $\mathrm{Li}_{0,33} \mathrm{MoO}_{3}$ was derived on the basis of tight-binding band calculations on the $\mathrm{Mo}_{6} \mathrm{O}_{24}$ chains that constitute $\mathrm{Li}_{033} \mathrm{MoO}_{3}$. The distortions of the $\mathrm{MoO}_{6}$ octahedra in $\mathrm{Li}_{0.33} \mathrm{MoO}_{3}$ were analyzed from the viewpoint of the $\mathrm{O}-\mathrm{Mo} \cdots \mathrm{O}$ bond alternation so as to deduce how the $\mathrm{t}_{2 \mathrm{~g}}$-level degeneracy of a regular $\mathrm{MoO}_{6}$ octahedron is lifted. We found this analysis to be a convenient way of understanding the nature of the bottom d-block bands of $\mathrm{Li}_{0.33} \mathrm{MoO}_{3}$. This study suggests that $\mathrm{Li}_{0.33} \mathrm{MoO}_{3}$ is a small band gap semiconductor.

Two classes of molybdenum oxide bronzes have been extensively studied because of their low-dimensional structural and electronic properties. The blue bronzes $\mathrm{A}_{0.3} \mathrm{MoO}_{3}(\mathrm{~A}=\mathrm{K}, \mathrm{Rb}, \mathrm{Tl})$ are two-dimensional (2D) in structure ${ }^{2}$ in that they are made up of metal-oxygen layers with composition $\mathrm{MoO}_{3}$. However, the blue bronzes are quasi-one-dimensional (1D) metals and, as a result, exhibit charge density wave (CDW) phenomena. ${ }^{3}$ The purple bronzes $\mathrm{A}_{0.9} \mathrm{Mo}_{6} \mathrm{O}_{17}(\mathrm{~A}=\mathrm{Na}, \mathrm{K})$ and $\mathrm{TlMo}_{6} \mathrm{O}_{17}$ consist of

(1) (a) Universitê de Paris Sud. (b) North Carolina State University.

(2) (a) Ghedira, M.; Chenavas, J.; Marezio, M.; Marcus, J. J. Solid State Chem. 1985, 57, 300. (b) Graham, J.; Wadsley, A. D. Acta Crystallogr. 1966, 20, 93. (c) Ganne, M.; Boumaza, A.; Dion, M.; Dumas, J. Mater. Res. Bull. 1985, 20, 1297. (d) Collins, B. T.; Ramanujachary, K. V.; Greenblatt, M.; Waszczak, J. V. Solid State Commun. 1985, 56, 1023

(3) (a) Schlenker, C.; Dumas, J. In Crystal Structures and Properties of Materials with Quasi-One-Dimensional Structures; Rouxel, J., Ed.; Reidel: Dordrecht, The Netherlands, 1986, p 135. (b) Moret, R. Pouget, J. P. Ibid, p 87 . (c) Whangbo, M.-H.; Schneemeyer, L. F. Inorg. Chem. 1986, 25, 2424. (d) Pouget, J. P.; Noguera, C.; Moudden, A. H.; Moret, R. J. Phys. (Les Ulis, Fr.) 1985, 46, 1731 metal-oxygen layers with composition $\mathrm{Mo}_{6} \mathrm{O}_{17}{ }^{4}$ Although these purple bronzes are $2 \mathrm{D}$ metals, they show CDW phenomena ${ }^{5}$ as well. In contrast, the lithium purple bronze $\mathrm{Li}_{0.9} \mathrm{Mo}_{6} \mathrm{O}_{17}$ differs

(4) (a) Vincent, H.; Ghedira, M.; Marcus, J.; Mercier, J.; Schlenker, C. J. Solid State Chem. 1983, 47, 113. (b) Gatehouse, B. M.; Lloyd, D. J.; Miskin, B. K. NBS Spec, Publ. (U.S.) 1972, No. 364, 15 . (c) Stephenson, N. C. Acta Crystallogr. 1966, 20, 59. (d) Greenblatt, M. Ramanujachary, K. V.; McCarroll, W. H.; Neifeld, R.; Waszczak, J V. J. Solid State Chem. 1985, 59, 149, (e) Ganne, M.; Dion, M. Boumaza, A.; Tournoux, M. Solid State Commun. 1986, 59, 137. (f) Ramanujachary, K. V.; Collins, B. T.; Greenblatt, M. Solid State Commun. 1986, 59, 647

(5) (a) Buder, R.; Devenyi, J.; Dumas, J.; Marcus, J.; Mercier, J.; Schlenker, C. J. Phys. (Les Ulis, Fr.) 1982, 43, L59. (b) Bervas, E. Cochrane, R. W.; Dumas, J.; Escribe-Filippini, C.; Marcus, J.; Schlenker, C. Lect. Notes Phys. 1985, 217, 144. (c) Dumas, J.; Bervas, E.; Marcus, J.; Salomon, D.; Schlenker, C. J. Magn. Magn. Mater. 1983, 31-34, 535. (d) Escribe-Filippini, C.; Konate, K.; Marcus, J.; Schlenker, C.; Almairac, R.; Ayroles, R.; Roucau, C. Philos. Mag. B 1984, 50, 321. (e) Whangbo, M.-H.; Canadell, E.; Schlenker, C. J. Am. Chem. Soc. 1987, 109, 6308. 